Recepción: 25 / 10 / 2017

Aceptación: 28 / 11 / 2017

Publicación: 15 / 12 / 2017

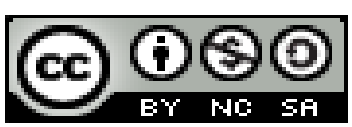

Ciencias de la Computación

Artículo de investigación

\title{
Formación de competencias desde la práctica profesional de ingenieros en ciencias informáticas
}
Training of competences from the professional practice of computer science engineers

Formação de competências da prática profissional de engenheiros de informática

Salvador González-Gómez ${ }^{\mathrm{I}}$ sgg.2020@gmail.com

Yasser Azán-Basallo II yazan@uci.cu

Yeneidys Alvarez-González III yalvarezg2014@gmail.com

Teresa Mina-Quiñonez ${ }^{\text {IV }}$ teresa_mina@hotmail.com

Marisol Morales-Martínez ${ }^{\mathrm{V}}$ marysolmoralesmartinez@gmail.com

Correspondencia:sgg.2020@gmail.com

\footnotetext{
${ }^{\text {I }}$ Master Universitario en Telemática y Redes de Telecomunicación, Ingeniero en Ciencias Informáticas, Universidad Técnica Luis Vargas Torres de Esmeraldas, Esmeraldas, Ecuador.

II Docente de la Universidad de las Ciencias Informáticas, La Habana, Cuba.

III Ingeniero en Ciencias Informáticas, Universidad Técnica Luis Vargas Torres de Esmeraldas, Esmeralda, Ecuador.

${ }^{\text {IV }}$ Magister en Administración de Empresas, Ingeniera en Sistemas Informáticos y de Computación, Universidad Técnica Luis Vargas Torres de Esmeraldas, Esmeraldas, Ecuador.

${ }^{\mathrm{V}}$ Master en Ciencias de la Educación Mención Educación de Adultos, Licenciado en Educación Especialidad Matemática, Universidad Técnica Luis Vargas Torres De Esmeraldas, Esmeraldas, Ecuador.
} 


\title{
Resumen
}

Se presenta una propuesta para la formación de competencias en la práctica profesional a estudiantes de la carrera de Ingeniería en Ciencias Informáticas. Se identifican la importancia que reviste en la actualidad para los egresados en ingeniería informática y los problemas actuales para la formación de las competencias en esta carrera. Se plantean competencias básicas y las formas de evaluación previstas para lograr los objetivos de formación de los estudiantes en la carrera. Este enfoque implica cambios en la concepción de esta disciplina y en la repercusión en el profesorado de la misma.

Palabras clave: ciencias informáticas; competencias; ingeniero; práctica profesional.

\begin{abstract}
A proposal for the formation of competences in professional practice is presented to students of the Computer Science Engineering career. It identifies the importance that currently holds for graduates in computer engineering and the current problems for the training of skills in this career. Basic competences and the forms of evaluation planned to achieve the objectives of training students in the race are considered. This approach implies changes in the conception of this discipline and in the impact on the teaching staff of it.
\end{abstract}

Keywords: computer science; competencies; engineer; professional practice.

\section{Resumo}

Uma proposta para a formação de competências na prática profissional é apresentada aos alunos da carreira de Engenharia da Computação. Identifica a importância que atualmente ocupa para os graduados em engenharia da computação e os problemas atuais para a formação de competências nesta carreira. Competências básicas e as formas de avaliação planejadas para alcançar os objetivos de treinar estudantes na corrida são consideradas. Esta abordagem implica mudanças na concepção desta disciplina e no impacto sobre o corpo docente da mesma.

Palavras chave: ciência da computação; competências; Engenheiro pratica profissional. 


\section{Introducción}

No cabe duda de la importancia y necesidad de educar en competencias. La complejidad del conocimiento, junto a la flexibilización y constante adaptación y actualización que promueven la Sociedad del Conocimiento, demandan una formación integral de los egresados de modo que adquieran competencias no sólo cognitivas, sino procedimentales y actitudinales que favorezcan una mejora de su proceso profesionalizado. En definitiva, requieren de un sistema basado en el desarrollo de competencias, pero ello no hace mención exclusiva a los métodos de enseñanzaaprendizaje, sino también a la evaluación de las mismas.

Tobón, señala que las competencias constituyen la base fundamental para orientar el currículo, la docencia, el aprendizaje y la evaluación desde un marco de calidad, buscando asegurar el aprendizaje de los estudiantes. A esto se añade que las competencias permiten, según Coll, identificar, seleccionar, caracterizar y organizar los aprendizajes del alumnado y por tanto, delimita también lo que debe enseñar el profesorado.

\section{¿Pero qué es la competencia?}

Por competencia se entiende según la COMISIÓN EUROPEA, es el "paquete multifuncional y transferible de conocimientos, destrezas y actitudes que todos los individuos necesitan para su realización y desarrollo personal, inclusión y empleo". Cano, publica que se puede entender como "una combinación de habilidades prácticas, conocimientos, motivación, valores éticos, actitudes, emociones y otros componentes sociales y de comportamiento que se movilizan conjuntamente para lograr una acción eficaz".

La competencia no se limita exclusivamente a la dimensión cognitiva, sino que presenta un carácter más global e integrado, recogiendo en su propia conceptualización dimensiones no cognitivas (habilidades, actitudes, valores y emociones), considerando además éstas competencias como fundamentales o imprescindibles, ya que nos permiten hacer frente a las exigencias de la vida en diferentes contextos. Suponiendo un proceso permanente de reflexión y evolutivo a lo largo de la vida. En este sentido, las competencias deben contribuir a obtener resultados de alto valor personal y social, ser aplicables y gozar de carácter superador. 


\section{¿Por qué apostar por la formación de competencias en la práctica profesional?}

Según lo publicado por Cano, en primer lugar nos hallamos inmersos en la sociedad del conocimiento. En los últimos años ha crecido exponencialmente la información que circula por la red. Por ello más que conocer ciertas informaciones que pueden dejar de ser válidas en un cierto tiempo, se hace necesario ser capaz de buscar la información pertinente a cada momento, ser capaz de seleccionarla (de entre un abanico de posibilidades), ser capaz de procesarla, tratarla, interpretarla y apropiarse de ella para generar el conocimiento necesario que nos permita resolver las situaciones que se nos presenten. Eso es precisamente lo que pretenden los diseños por competencias: que las personas desarrollen capacidades amplias, que les permitan aprender y desaprender, a lo largo de toda su vida para adecuarse a situaciones cambiantes. Es posible que no ocupemos el mismo puesto de trabajo toda la vida. Quizá no desempeñemos ni siquiera la misma profesión. Necesitamos conocimientos, habilidades y actitudes que nos faciliten esa flexibilidad que se hará imprescindible.

En segundo lugar y ligado al cambio acelerado del saber, hallamos la complejidad: el conocimiento es cada vez más complejo, obedeciendo a una lógica posmoderna que nos cuesta articular porque equiparamos complejidad y complicación. Las clásicas divisiones entre asignaturas o las clasificaciones de los saberes parecen no servir en el contexto actual. Hay que eliminar la fragmentación y apostar por un conocimiento integrado que, como sugiere Morín, supere la superespecialización y el reduccionismo que aísla y separa.

Por la anterior afirmación, la disciplina práctica profesional, para las carreras informáticas, históricamente se ha propuesto garantizar el espacio de integración de todo el conocimiento asimilado en el proceso de aprendizaje de los estudiantes, a través de actividades prácticas de investigación y producción en el desarrollo de sistemas informáticos y de software. En la Universidad de las Ciencias Informáticas a todo esto se le agregó la formación directamente orientada a la actividad de producción y desarrollo por medio de la asimilación, en cursos, de los conocimientos y habilidades necesarios para desempeñarse en los distintos modos de actuación expresados en el modelo del profesional. A partir de la introducción del modelo de integración Docencia - Producción - Investigación, se crean las condiciones para que la Práctica Profesional 
pueda concretar los objetivos y el diseño que la convierten verdaderamente en la Disciplina Principal Integradora del plan de estudio de la carrera.

Con esta disciplina además lograr dotar en el egreso de ingenieros, ciertas capacidades y atributos personales que los hagan aptos para insertarse al trabajo productivo en forma rápida y eficaz y acercar el entorno laboral al aula.

\section{Materiales y métodos. Las competencias básicas}

A diferencia de carreras como Arquitectura, Medicina y Derecho, entre otras, el ámbito de trabajo de la Ingeniería es muy amplio en cuanto a áreas de desempeño y poco predecible. Los ingenieros pueden trabajar en proyectos y diseños muy variados, gestión, operaciones, desarrollo, ventas, etc.

Por esta razón, identificar las competencias más necesarias para un contexto laboral amplio y cambiante es difícil. Deben combinarse competencias técnicas, como las competencias que corresponden a las tareas anteriormente indicadas, con competencias más generales y adaptativas. Por otra parte, precisar el alcance que esas competencias deberían tener al egreso, que es el punto hasta donde llega el ámbito de acción de la universidad en el pregrado, es otra dificultad.

Otro problema identificado por Letelier, es el peso curricular que tiene el conocimiento transferido, así como la potencialidad de éste para generar habilidades y competencias. El conocimiento asociado a las ciencias físico-matemáticas proporciona un amplio y sólido fundamento para el desarrollo de las tecnologías en que se apoya el desempeño profesional de los ingenieros. Varias competencias profesionales, tanto técnicas o especializadas como generales, son en alta medida dependientes, influidas o inducidas por las tecnologías.

- Competencias técnicas: En esta competencia es donde se evidencia el trabajo por roles en los proyectos de investigación que se les orienta en la actividad laboral en los centros productivos de la universidad. Los roles previstos a trabajar por lo estudiantes son:

○ Programador

○ Diseñador de Base de Datos

○ Diseñador de Pruebas 

- Analista de sistema

- Analista de Procesos de Negocio

- Administrador de Base de Datos

○ Soporte de software y hardware

- Auto-aprendizaje: Capacidad de mantenerse actualizado y de desarrollar las capacidades y atributos que el entorno laboral demanda.

- Ética profesional: Capacidad de identificar, analizar y resolver problemas de ética profesional.

- Comunicación: Capacidad de informar, de recibir información y de persuadir.

- Trabajo en equipo: Capacidad de asumir responsabilidades en trabajo grupal con un fin común.

- Innovación: Capacidad de proponer y desarrollar nuevas y mejores formas de realizar tareas profesionales.

- Emprendimiento: Capacidad de desarrollar iniciativas de carácter económico, social y/o cultural, a través de realización de proyectos, que requieren de toma de decisiones, asumir riesgos y de liderazgo.

\section{Resultados. La evaluación por competencia desde la práctica profesional}

Con respecto a la evaluación en la educación superior, se toma como puntos de partida las siguientes consideraciones:

- La evaluación no puede limitarse a la calificación (sino que ésta es un subconjunto de la evaluación); no puede centrarse en el recuerdo y la repetición de información (sino que se deben de evaluar habilidades cognitivas de orden superior) y que no puede limitarse a pruebas de "lápiz y papel”, sino que se requieren instrumentos complejos y variados.

- La evaluación por competencias nos obliga a utilizar una diversidad de instrumentos y a implicar a diferentes agentes. Tenemos que tomar muestras de las ejecuciones de los 
alumnos y utilizar la observación como estrategia de recogida de información sistemática. Ésta puede acompañarse de registros cerrados (lista de cheque o escalas) o de registros abiertos y puede hacerse por parte del profesorado, por parte de los compañeros o por parte del propio estudiante (o por todos ellos, en un modelo de evaluación de $360^{\circ}$ ), pero en cualquier caso debe proporcionar información sobre la progresión en el desarrollo de la competencia y sugerir caminos de mejora.

- La evaluación ha de hacer más conscientes a los estudiantes de cuál es su nivel de competencias, de cómo resuelven las tareas y de qué puntos fuertes deben potenciar y qué puntos débiles deben corregir para enfrentarse a situaciones de aprendizaje futuras. Este proceso de autorregulación, tratado, entre otros, por va a ser esencial para seguir aprendiendo a lo largo de toda la vida y constituye en sí mismo, una competencia clave.

A partir de las consideraciones anteriores, se definió el siguiente sistema de evaluación que miden las competencias básicas definidas:

- Realizar presentaciones de trabajos finales, que deben asociarse documentaciones de respaldo: informes técnicos, científicos y presentaciones que apoyen las exposiciones de la información. Las evaluaciones, por tanto, tendrán en cuenta la calidad de esta documentación en el momento de calificar. Se tendrán en cuenta además, los resultados en el ejercicio práctico, dígase productos o soluciones obtenidas y cumplimiento de las tareas asignadas en los proyectos, una vez vinculados a los mismos. Además se harán las presentaciones en equipos.

- Evaluar las tareas asignadas mensualmente a cada estudiante individualmente. Las tareas, en los casos que así procedan, deben tener determinados aseguramientos instructivos como: teoría, bibliografía, ejemplos, entre otros. Deben tener además un resultado medible, los cuales pueden ser: artefactos, documentos, informes, aplicación, código fuente, etc. Los resultados de estas tareas deben ser presentados en talleres, seminarios o reuniones de proyectos.

- La evaluación final de las asignaturas se realiza a través de la discusión de un informe final, donde se presenten los resultados de las tareas realizadas durante la asignatura en todo el semestre. 
- Los tribunales que evalúan la defensa de los informes finales se organizan por el Jefe de Departamento de la facultad de conjunto con los centros productivos. La nota final del estudiante la otorga el profesor de la asignatura a partir de las evaluaciones mensuales y el criterio de los tutores en caso de tenerlos.

\section{Conclusiones}

La formación por competencias desde la práctica profesional permite la integración de los conocimientos, habilidades y valores identificados en la carrera de ingeniería en ciencias informáticas. Promueve que el estudiante adquiera con la práctica, las competencias que requiere un egresado de esta carrera, para poder enfrentar las distintas necesidades existentes en las empresas, entidades u organizaciones donde se le requiera.

\section{Referencia Bibliográfica}

D. Allen, Ed. "La evaluación de los aprendizajes de los estudiantes. Una herramienta para el desarrollo profesional de los docentes. Barcelona, Paidós“. Edition. City. Vol. pp. Number of 2000. DOI:

K. Bain. "Lo que hacen los mejores profesores universitarios. Valencia: Publicacions Universitat de València”. Edition. City. Vol. pp. Number of 2006. DOI:

M. Boekaerts, P. Pintrich, y M. Zeidner. "Handbook of self-regulation. Nueva York: Academic Press”. Edition. City. Vol. pp. Number of 2000. DOI:

G.E. Cano. "La evaluación por competencias en la educación superior". Edition. City. Vol. pp. Number of 2011. DOI:

C. COLL. "Los enfoques curriculares basados en competencias y el sentido del aprendizaje escolar. In Conferencia magistral presentada en el $\mathrm{X}$ Congreso Nacional de Investigación Educativa, Veracruz, México: COMIE (www.comie. org. mmx)". Edition. City. Vol. pp. Number of 2009. DOI:

F. Dochy, M. Segers, y S. Dierick. "Nuevas Vías de Aprendizaje y Enseñanza y sus Consecuencias: Una Nueva Era de Evaluación. Boletín de la Red Estatal de Docencia Universitaria, 2, 2, 13-29".Edition. City. Vol. pp. Number of 2002. DOI: 
C. EUROPEA. "Competencias "claves" para un aprendizaje a lo largo de la vida. Un marco de referencia europeo". Edition. City. Vol. pp. Number of 2004. DOI:

M.S. Letelier y otros. "SISTEMA DE COMPETENCIAS SUSTENTABLES PARA EL DESEMPEÑO PROFESIONAL EN INGENIERÍA". Rev. Fac. Ing.-Univ. Tarapacá. Edition. City. Vol. 13, pp. Number of 91-96. 2005. DOI:

R. McDonald y otros. "Nuevas perspectivas sobre la evaluación. Boletín Cinterfor, 149, 4172".Edition. City. Vol. pp. Number of 2000. DOI:

E. Morin. "Los siete saberes necesarios para la educación del futuro. Barcelona: Seix Barral". Edition. City. Vol. pp. Number of 2001. DOI:

O.C.D.E. "Definition and Selecction of Competencies: Theoretical and Conceptual Foundations (DeSeCo). Disponible en URL: http: //www.oecd.org/education/skillsbeyondschool/41529556.pdf". Edition. City. Vol. pp. Number of 2001. DOI:

Á. PÉREZ. "La naturaleza de las “competencias básicas” y sus implicaciones pedagógicas. Características principales de las competencias básicas. “.Cuadernos de educación de Cantabria. Edition. City. Vol. 1, pp. Number of 13-16. 2007. DOI:

S. TOBÓN. "Aspectos básicos de la formación basada en competencias. Talca: Proyecto Mesesup". Edition. City. Vol. pp. Number of 2006. DOI:

A.d.C.-H. Tolino. "Evaluación de competencias en el grado de educación social". Revista de Ciencias Humanas y Sociales. Edition. City. Vol. pp. Number of 750-767. 2016. DOI: 